\title{
A revista e seus elos
}

\author{
Harley E. A. Bicas, Cristina Muccioli, Mauro Goldchmit,Mauro S. O. Campos, \\ Paulo E. Correa Dantas, Samir Jacob Bechara, Vital Paulino Costa
}

Apesar de já antes mencionado, nunca é demais voltar ao assunto da importância dos Conselheiros Editoriais numa revista científica. Obviamente, ela se faz de outras pessoas, serviços e colaborações, imprescindíveis tanto quanto aqueles: autores, como provedores do material substantivo da revista; secretárias, dando andamento (burocrático e diplomático) às idas e vindas dos trabalhos em suas fases intermediárias; normatizadoras, adequando as apresentações; revisores, dando à revista sua forma final e formatação; impressores, dando realidade às publicações; editores, supervisionando o processo e zelando pela qualidade de tudo. Mas todos, de um modo ou de outro, têm seus pagamentos. Os primeiros (autores) recebendo o aval sobre que aquilo que pensaram e realizaram merece registro, difusão e perenização. Os últimos (editores) por terem reconhecida, explicitamente, a condição de delegados da Oftalmologia brasileira para o distintivo exercício de conduzir esse trabalho "cartorial" de registro de escrituras, dando legitimidade e posse a idéias inovadoras; e o de incentivar a difusão de conhecimentos. Os intermediários auferindo, em espécie, pelos digníssimos serviços prestados.

Mas além desses todos há os que, também "vestindo a camisa" dos A.B.O., distinguem-se por trabalhos importantíssimos e que, pela própria condição do que fazem, devem ser protegidos pelo anonimato. São os que analisam os artigos, detectam falhas, aconselham autores, recomendam modificações, emitem julgamentos de recusa ou aceitação das respectivas publicações. É por eles que se garante o padrão da revista; é com eles que se chega a melhorar a qualidade de cada artigo. A eles devem caber os agradecimentos de leitores, pelo texto depurado de impropriedades; a eles, igualmente, a reverência dos autores, pelas sugestões recebidas. Já vai longe o tempo em que se considerava "amigo" o analista editorial condescendente com a publicação, independentemente da qualidade que ela tivesse. Hoje, ao contrário, já se considera o Conselheiro Editorial tanto mais favoravelmente quanto maiores as ajudas encaminhadas, ainda que elas impliquem extensas remodelações do trabalho (e justamente por isso são importantes).

Os Arquivos Brasileiros de Oftalmologia inovam ao permitir que esse trabalho anônimo possa vir a ser publicamente reconhecido, se o conselheiro assim o desejar. Oferece-se-lhe a opção de ter seu nome vinculado ao do trabalho, como seu analista, ao fim do processo editorial. (Em todos os casos o sigilo é mantido, tanto sobre os nomes dos autores para os que analisam os trabalhos como os destes para aqueles.) De fato, pela leitura atentamente realizada com o intuito de revisão, pela análise percuciente, pelas variadas sugestões de melhoria, freqüentemente aceitas pelos autores e incorporadas ao trabalho, os revisores tornam-se como que colaboradores fundamentais da publicação, quase seus co-autores (possivelmente, em muitos casos, com a compreensão do conjunto até mais aguçada e a participação mais comprometida do que alguns dos muitos coautores "oficiais" de um trabalho). É justo que possam ter os devidos reconhecimentos. Mas também é óbvio que isso implica aval à publicação, nem sempre aceitável. Talvez por isso, talvez pelo constrangimento de se identificar aos autores depois de críticas eventualmente duras e de necessários rigores, muitos ainda optem pelo anonimato.

Chega o momento de desvendar as importantíssimas ajudas, revelar os trabalhos realizados -- ainda que sem identificar-lhes as circunstâncias especificas - e fazer os apropriados e justos agradecimentos. Ultimamente, aliás, os Editores têm optado por exigir análises ainda mais extensas e diversificadas: cada trabalho submetido aos Arquivos Brasileiros de Oftalmologia tem sido distribuído, quase sempre e já no início do processo de análise editorial, a três examinadores. Daí a conveniência de chamamento de outras pessoas para essa função, além das já previstas e preferencialmente acionadas (na lista dos Conselheiros Editoriais). Esses conselheiros "ad hoc" que tanto contribuíram para o engrandecimento dos A.B.O. no ano de 2003 e aos quais apresentamos os nossos agradecimentos e os da Oftalmologia nacional são:
A. Duarte
Acácio Alves de Souza Lima Filho
Acácio Muralha Neto
Adamo Lui Netto
Adriana Berezovsky
Adriana dos Santos Forseto
Alexandre Chater Taleb
Alfredo Tranjan Neto
Amélia Kamegasawa
Ana Estela B. P. P. Sant' anna
André Barbosa Castelo Branco
André Correia Maia de Carvalho
André Marcelo Vieira Gomes
Andréa Araújo Zin
Ângela Maria de Queiroz Vieira
Arnaldo Furman Bordon
Breno Barth
Carlos Akira Omi
Carlos Eduardo Leite Arieta
Carlos Fumiaki Uesugui
Carlos Roberto Neufeld
Carlos Rubens L. de Figueiredo 
Carlos Teixeira Brandt

Carmo Mandia Jr

Célia Regina Nakanami

César Lipener

Christiane Rolim de Moura

Christine Mae Morello Abbud

Cinthia Oyama

Cláudia Maria Francesconi

Cláudio Alberto M. Silveira

Clélia Maria Erwenne

Consuelo Bueno D. Adam

Davi Araf

Denise de Vuono Chinzon

Denise Fornazari de Oliveira

Diane R. Marinho

Edison João Geraissate Filho

Edméa Rita Temporini

Edmundo de Almeida Frota Sobrinho

Edson Shizuo Mori

Eduardo Ferrari Marback

Eduardo Jorge Carneiro Soares

Eduardo Marcelo Moron de Andrade

Eduardo Melani Rocha

Eduardo Minelli

Eduardo Sone Soriano

Elcio Hideo Sato

Eliana Aparecida Forno

Elisabeto Ribeiro Gonçalves

Emerson Francisco P. Neves

Eric Pinheiro de Andrade

Eurípedes da Mota Moura

Fábio Ejzebaum

Fábio Henrique C. Casanova

Fausto Uno

Fernando César Abib

Flávio Jaime da Rocha

Flávio Rocha Lima Paranhos

Francisco de Assis Cordeiro Barbosa

Francisco Eduardo Lopes de Lima

Francisco Porfírio Neto Junior

Francisco Valter da Justa Freitas

Geraldo de Barros Ribeiro

Helcio José Fortuna Bessa

Helia Soares Angotti

Henrique S. Kikuta

Italo Mundialino Marcon

Jair Giampani Jr

Joao Alberto Holanda de Freitas

Joao Carlos de Miranda Goncalves

Joaquim Marinho de Queiróz Jr

John Helal Jr

José Américo Bonatti

Jose Augusto Cardillo

José Carlos Eudes Carani

José Paulo Cabral Vasconcellos
José Vital Filho

José Wilson Cursino

Joyce Hisae Yamamoto

Juliana Maria F. Sallum

Keila Miriam Monteiro de Cavalho

Kozo Nakano

Laurentino Biccas Neto

Leiria de Andrade Neto

Leonardo Akaishi

Liana Maria O. Ventura

Lucia Miriam D. Lucci

Luciana Peixoto Finamor

Luciene Barbosa de Sousa

Luciene Chaves Fernandes

Luís Carlos Ferreira de Sá

Luís Eduardo Morato R. Carvalho

Luiz Alberto Soares Melo Jr

Luiz Antonio Vieira

Magno Antonio Ferreira

Marcelo Carvalho Ventura

Marcelo Francisco G. Vadas

Marcia Beatriz Tartarella

Marcia Keiko U. Tabuse

Márcia Regina Issa Salomão

Marco Aurélio Lana-Peixoto

Marco César Helena

Marcos Carvalho da Cunha

Marcos Wilson Sampaio

Maria Aparecida Onuki Haddad

Maria Auxiliadora M. F. Sibinelli

Maria Carmen Santos Torres

Maria Cristina Martins

Maria de Lourdes M.M. Villas Boas

Maria Kiyoko Oyamada

Maria Rosa Bet de Moraes

Marilisa Nano Costa

Maristela Amaral Palazzi

Mariza Aparecida Polati

Mariza Toledo de Abreu

Marta Beatriz F. Sartori

Martha Maria M. Chojniak

Mauricio Bastos Pereira

Mauricio Della Paolera

Mauro Nishi

Mauro Waiswol

Miguel Ângelo Gontijo Álvares

Milton Massato Hida

Milton S. Yogi

Miriam Skaf

Moacyr P. Rigueiro

Nelson Alexandre Sabrosa

Newton Kara-José Jr

Nilo Holzchuh

Nilson de Mello E Oliveira

Nilva Simeren Bueno Moraes 
Niro Kasahara

Norma Allemann

Norma Helen Medina

Núbia Cristina F. Maia

Paiva Gonçalves Filho

Paiva Gonçalves Neto

Paola Zucchi

Patrícia Ioschpe Gus

Paulo André Polisuk

Paulo César Silva Fontes

Paulo Gelman Vandergorn

Paulo Gilberto Jorge Fadel

Paulo Góis Manso

Paulo Henrique de Ávila Morales

Regina Aidar Menon Nosé

Renato Ambrosio Jr

Renato Giovedi

Renato Luiz Gonzaga

Ricardo Belfort

Ricardo Morschbacher

Ricardo Themudo L. Waetge

Roberto Freda

Roberto Lauande Pimentel

Roberto Mitiaki Endo
Rodrigo Jorge

Rosa Maria Graziano

Rosana Nogueira Pires da Cunha

Rosane da Cruz Ferreira

Rui Barroso Schimiti

Ruth M. Santo

Samuel Rymer

Seije Hayashi

Sérgio Vanetti Burnier

Sidney Julio de Faria E. Sousa

Silvia Veitzman

Teruo Aihara

Tomas F. Scalamandré Mendonça

Vera Lúcia D. Monte Mascaro

Vera Regina C. Castanheira

Virgílio Centurion

Wagner Zacharias

Walter Takahashi

Walton Nosé

Wesley Ribeiro Campos

Wilmar Roberto Silvino

Wilson de Freitas

Yara Michelacci

Zélia Maria da Silva Corrêa

\section{Simpósio Internacional da Santa Casa de São Paulo}

\section{3 a 5 de Junho de 2004 \\ Centro de Convençóes Frei Caneca - SP}

INFORMAÇÕES: JDEComunicaçãoe Eventos

Tels.: (1 1) 287-9378 / 289-4301

Fax: (1 1 ) 288-8157

E-mail: jdecomev@uol.com.br 